\title{
Comparison of the paediatric Yorkhill malnutrition score (PYMS) with other paediatric screening/assessment methods
}

\author{
K. Gerasimidis, O. Keane ${ }^{1}$, I. Macleod ${ }^{1}$, E. Buchanan ${ }^{1}$, P. $\operatorname{Mcgrogan}^{1}$, G Stewart ${ }^{1}$, A. Maclean ${ }^{1}$, \\ D. F. Flynn ${ }^{1}$ and C. M. Wright ${ }^{2}$ \\ ${ }^{1}$ Women and Children Directorate, NHS Greater Glasgow and Clyde, Glasgow, UK and ${ }^{2}$ Faculty of Medicine, University of \\ Glasgow, Glasgow, UK
}

All inpatients should be screened for malnutrition ${ }^{(1)}$ but validated paediatric tools for use by nursing staff are scarce. The Paediatric Yorkhill Malnutrition score (PYMS) was developed based on ESPEN guidelines ${ }^{(2)}$. Its development, performance ${ }^{(3)}$, criterion validity, inter-rater reliability ${ }^{(4)}$ and impact on clinical practice ${ }^{(5)}$ have been assessed. This study compared the PYMS with other similar paediatric tools.

Two research dietitians screened malnutrition using the PYMS, the Screening Tool for the Assessment of Malnutrition in Paediatrics $(\text { STAMP) })^{(6)}$ and the paediatric Subjective Global Nutritional Assessment (SGNA) ${ }^{(7)}$ in inpatients (1-16 years) from a tertiary paediatric $(n=225)$ and district general hospital $(n=22)$.

Two hundred and forty seven children consented to the study. The prevalence of malnutrition varied between the different tools. Compared to PYMS, STAMP identified more patients as being at risk, while SGNA identified fewer (Table 1).

\begin{tabular}{lccc}
\hline \multicolumn{5}{c}{ Table 1 } & \\
\hline Pigh risk & PYMS $(\%)$ & STAMP $(\%)$ & SGNA $(\%)$ \\
Medium risk & 21 & 28.7 & 1.6 \\
\hline
\end{tabular}

Eighty percent (198/247) of the patients were classified at the same risk of malnutrition between STAMP and PYMS and $81 \%$ (199/ $247)$ between SGNA and PYMS (Table 2). The agreement between STAMP and PYMS was moderate $(\kappa=0.4795 \%$ CI [0.34-0.61]) and between SGNA and PYMS slight $(\kappa=0.1295 \%$ CI $[-0.11-0.34])$. PYMS identified all the children who screened at risk by SGNA but only $52 \%$ of those screened at risk by STAMP (Table 2). Likewise $20 \%$ and $9 \%$ of the patients screened at low risk using SGNA and STAMP were classified at risk by PYMS (Table 2).

Table 2

\begin{tabular}{llccrrr}
\hline & \multicolumn{2}{c}{ STAMP } & & \multicolumn{2}{c}{ SGNA } \\
\cline { 3 - 4 } & & No risk* & Risk & & No risk & Risk \\
\hline PYMS & No risk* & $161(91 \%)$ & $34(48 \%)$ & & $195(80 \%)$ & $0(0 \%)$ \\
& Risk & $15(9 \%)$ & $37(52 \%)$ & & $48(20 \%)$ & $4(100 \%)$ \\
\hline
\end{tabular}

*Low and medium risk combined.

STAMP identified a greater percentage of inpatients as being at medium or high risk, while PYMS identified a more manageable proportion. PYMS agreed poorly with SGNA which identifies malnourished rather than patients at risk of developing malnutrition. Comparison against dietetic assessment is needed to explore which screening tool has better diagnostic validity.

1. NHS Quality Improvement Scotland (2003) Food, Fluid and Nutritional Care in Hospitals.

2. Kondrup J, Allison SP, Elia M et al. (2003) Clin Nutr 22, 415-421.

3. Gerasimidis K, Macleod I, McGrogan P et al. (2009a) BSPGHAN Annual Winter Meeting 2009

4. Gerasimidis K, Keane O, Macleod I et al. (2009b) ESPGHAN Annual Meeting 2009 (in press).

5. Macleod I, Gerasimidis K, Purcell O et al. (2009) ESPGHAN Annual Meeting 2009 (in press)

6. McCarthy H, McNulty H, Dixon M et al. (2008) JHum Nutr Diet 21, 395-396.

7. Secker DJ \& Jeejeebhoy KN (2007) Am J Clin Nutr 85, 1083-1089. 